Bangl. J. Vet. Med. (2008). 6 (1): 107-114

\title{
EFFICACY OF SOME INDIGENOUS PLANTS IN CONTROLLING VECTOR SNAILS OF TREMATODE PARASITES OF MEDICAL AND VETERINARY IMPORTANCE
}

\author{
I. S. Shanta*, Anisuzzaman, U. K. Mohanta, T. Farjana and M. M. H. Mondal
}

Department of Parasitology, Faculty of Veterinary Science, Bangladesh Agricultural University, Mymensingh-2202, Bangladesh

*Corresponding author's e-mail address: shantabau@yahoo.com

\begin{abstract}
To study molluscicidal effects of some indigenous plants such as Dhol Kalmi (Ipomoea fistulosa), Lantana (Lantana camara), Rakta-karabi (Nerium indicum), Polash (Butea frondosa), Mohavringoraj (Wedelia calandulacea), Nishinda (Vitex negundo), Bishkatali (Polygonum hydropiper), Kalmi, (Ipomoea aquatica), Haicha ( Alternanthera sessilis) and Shaora (Streblus asper) were used as dusts, aquatic, ethanol and methanol extracts against Lymnaea auricularia, Lymnaea luteola and Indoplanorbis exustus to give in vitro trial from January 2006 to December 2006 in the Department of Parasitology, Bangladesh Agricultural University, Mymensingh. All the plants in all preparations were more or less effective against the treated snails. In this study, $\mathrm{LD}_{50}$, Min. $\mathrm{C}_{\mathrm{s} 100}$ and mortality percentage of fish (Gutum, Letidocephalus guntea, Family: Cobitidae) were determined using dust of the selected plants. $\mathrm{LD}_{50}$ was minimum in case of Haicha $(14.3 \mathrm{mg} / \mathrm{L})$ followed by rakta-karabi $(29.25 \mathrm{mg} / \mathrm{L})$, bishkatali $(46.88 \mathrm{mg} / \mathrm{L})$, kalmi $(154.69 \mathrm{mg} / \mathrm{L})$, lantana $(234 \mathrm{mg} / \mathrm{L})$ etc. Min.C s100 $_{\text {was }}$ minimum in case of rakta-karabi $(0.008 \%)$ followed by haicha (0.03\%), lantana (0.06\%), dholkalmi (0.25\%) and kalmi $(0.25 \%)$. Among the plants, lantana, haicha and kalmi had no lethal effect on fish at Min.C $\mathrm{C}_{\mathrm{s} 100}$. Shaora also had no toxic effect on fish but its both $\mathrm{LD}_{50}(453.13 \mathrm{mg} / \mathrm{L})$ and Min.C $\mathrm{C}_{\mathrm{s} 100}(1 \%)$ were very high. Present study suggests that dust of haicha, lantana and kalmi may be used @ 0.03\%, 0.06\% and 0.25\% respectively to control snails in field level.
\end{abstract}

Key words: Indigenous plants, Lymnaea auricularia, Lymnaea luteola, Indoplanorbis exustus, fish

\section{INTRODUCTION}

Snails act as intermediate hosts of various trematode parasites. These parasites have adverse effects on the health and productivity of livestock and poultry. They are associated with economic losses in terms of morbidity, mortality, treatment cost and condemnation of carcass or part of carcass. Different types of anthelmintic medications are commonly used to control these parasites. But the control of vector snails is the best way to control trematode infections. Various types of chemical compounds such as calcium cyanide, sodium pentochlorophenate and N-tritylmorpholine are being used to control vector snails in different countries. But these are not so much environmentally friendly. Calcium cyanide and sodium pentochlorophenate cause dermatitis in man and animal. They kill aquatic plants and animals including fishes. Besides, calcium cyanide and sodium pentochlorophenate are rapidly inactivated by the ultraviolet ray and N-tritylmorpholine losses its activity with the change of $\mathrm{pH}$ (Malek and Cheng, 1974). Countries like India, China, Brazil etc. are conducting research to control vector snails of trematode parasites by using plant products. In Bangladesh, a preliminary survey has also been conducted to screen the plants having molluscidal efficacy on Indoplanorbis exustus (Sultana et al. 2004). Besides, a brief review of relevant literatures of other countries shows that exudate, bark and aqueous extract of leaf of Holde-karabi (Thevetia paruviana) kills Lymnaea acuminata and Indoplanorbis exustus (Singh and Singh 2001). Aqueous extract of neem fruits (Azadirachta indica) kills 100\% snails, Indoplanorbis exustus at $4 \%$ concentration within 12 hours. Similarly aqueous extract of leaves lantana (Lantana camara ) kills $100 \%$ snails at $0.05 \%, 0.1 \%$ and $0.2 \%$ concentration within 24,12 and 6 hours (Pal et al 2002) respectively. Wang et al (2006) evaluated the molluscicidal activities N-butanol extracts and water extracts of Nerium indicum. 
The $\mathrm{LD}_{50}$ value of the water extract of $N$. indicum was as low as $13.2 \mathrm{mg} / \mathrm{L}$. Singh and Singh et al. (2005) reported the mortality caused by the aqueous extract of latex of Thevetia peruviana, Alstonia scholaris and Euphorbia pulcherrima against two harmful freshwater snails, Lymnaea acuminata and Indoplanorbis exustus. Latices of all the plants at high doses were also lethal to freshwater fish Channa punctatus, but within $24 \mathrm{~h}, \mathrm{LC}_{90}$ of snail L. acuminata did not cause any mortality to fishes in a mixed population of snail and fish. By considering these points, the research work was furnished to screen indigenous plants to determine molluscicidal efficacy and to detect effect on fish.

\section{MATERIALS AND METHODS}

\section{Plant materials}

Fresh leaves of Dhol Kalmi (Ipomoea fistulosa, Family: Convolvulaceae), Lantana (Lantana camara, Family: Verbenaceae), Rakta-karabi (Nerium indicum, Family: Apocynaceae), Polash (Butea frondosa, Family: Leguminaceae), Mohavringoraj (Wedelia calandulacea, Family: Asteraceae), Nishinda (Vitex negundo, Family: Verbenaceae), Bishkatali (Polygonum hydropiper, Family: Polygonaceae ), Kalmi, (Ipomoea aquatica, Family: Convolvulaceae ), Haicha ( Alternanthera sessilis, Family: Amaranthaceae ) and Shaora (Streblus asper Lour., Family:Moraceae) were collected from Bangladesh Agricultural University (BAU) campus and its surrounding areas. The leaves were dried separately in a shaded place, pulverized with a pastle and mortar and sieved through a $25 \mathrm{~mm}$ mesh diameter sieve, and stored in airtight containers for further use.

\section{Preparation of plant extracts}

Extracts of plant materials were made by dissolving the pulverized leaves in methanol, ethanol and water. To do this, $10 \mathrm{gms}$ of each pulverized plant materials were soaked in $100 \mathrm{ml}$ of methanol, ethanol and water in separate glass beakers, stirred thoroughly for 30 minutes by using a magnetic stirrer at 200 rpm and kept for 24 hours to have the extracts. After that, each of the materials were filtered through a fine cloth and then through a filter paper. The filtrates were then taken separately into round bottom flasks and condensed by evaporating the solvents (methanol, ethanol and water) in water bath at 50 to $80^{\circ} \mathrm{C}$ until to get $10 \mathrm{ml}$ final extracts and stored in refrigerator as stock materials for molluscicidal screening.

\section{Test solutions}

Solutions of 1, 2 and 4\% strength were prepared by mixing the stock solutions in distilled water.

\section{Collection and maintenance of snails}

Indoplanorbis exustus, Lymnaea luteola and L. auricularia snails were collected from natural habitats and reared in the laboratory in glass aquaria and/or plastic containers by following appropriate technique (Malek and Cheng, 1974).

\section{Bioassay procedure}

For each assay, 10 snails of particular species were taken in large petridishes. Snails were submerged with the help of polyester net into the methanol, ethanol and water extracts of the plant leaves at different concentrations (1, 2 and 4\%). For control study, snails were treated with distilled water, $4 \%$ methanol and ethanol separately. Snails were examined at 6,12 and 24 hours after the treatment to detect the mortality by close inspection. Besides, snails were treated with dust of the fore-said plants in same manner. Fishes (Gutum, Letidocephalus guntea, Family: Cobitidae) were treated with dust of the tested plants @ Min. $\mathrm{C}_{100}$ in same way to determine the effect of these plant on fishes (Fig. 1). 


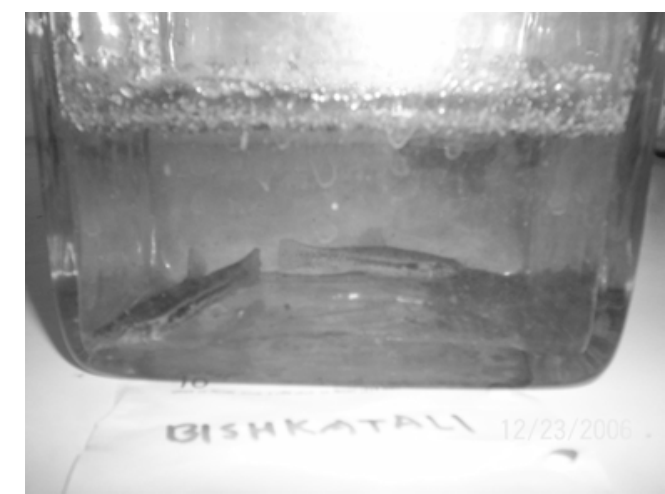

Fig. 1. Trial on fish (Gutum, Letidocephalus guntea).

\section{Statistical analysis}

For Complete Randomized Block Design (CRBD), data were subjected to ANOVA with 3 replicates. Effects of the different concentrations of plant extracts were determined by SAS procedure. Student t-test was used to determine the significance among the different variables (Steel and Torrie, 1980). Significant differences among the mean values of the same variable were determined by DMRT (Duncan's Multiple Range Test).

\section{RESULTS AND DISCUSSION}

\section{Effects on I. exustus}

Methanol extract of nishinda was the most effective against $I$. exustus, which caused death of $100 \%$ snails within 6 hours at all (1, 2 and 4\%) concentrations used. Mthanol extract of dhol kalmi, lantana, rakta-karabi, mohavingoraj, bishkatali and haicha killed $100 \%$ snails treated, by 24 hours at 1, 2 and 4\% concentrations. Two per cent and 4\% ethanol extracts of mohavingoraj, nishinda, rakta-karabi, dhol kalmi and haicha killed 100\% snails (I. exustus) within 6 hours. Water extract of 1, 2 and 4\% rakta-karabi and 4\% kalmi showed the highest efficacy (100\%) followed by $4 \%$ mohavingoraj (90\%) against $I$. exustus within 6 hours. Lantana, kalmi and mohavingoraj killed 100\% snails treated, at 2 and $4 \%$ concentrations by 24 hours (Table 1). Similar studies were conducted wtih Nerium indicum. Wang et al. (2006) evaluated the molluscicidal activities of N-butanol extract and water extract of Nerium indicum and found that $\mathrm{LD}_{50}$ value of water extract of Nerium indicum was 13.2 $\mathrm{mg} / \mathrm{L}$. Singh and Singh et al. (2005) observed that aqueous extract and latex of Thevetia peruviana, Alstonia scholaris and Euphorbia pulcherrima killed Indoplanorbis exustus. Exudate, bark and aqueous extract of leaf of Holde-karabi (Thevetia paruviana) killed Lymnaea acuminata and Indoplanorbis exustus (Singh et al., 2005). Extract of henna seed was effective against Lymnaea acuminata and Indoplanorbis exustus (Amrita et al., 2001). Aqueous extract of neem fruits (Azadirachta indica) killed 100\% snails (Indoplanorbis exustus) at $0.4 \%$ concentration within 12 hours. Similarly aqueous extract of leaves lantana (Lantana camara) killed 100\% snails at $0.05 \%, 0.1 \%$ and $0.2 \%$ concentration within 24, 12 and 6 hours respectively (Pal et al 2002). Mostafa et al (2005a) reported molluscicidal efficacy of Anagalis arvensis and Calendula micrantha against Biomphalaria alexadrina, Physa acuta, Planorbis planorbis, Helisoma duryi and Melanoides tuberculata. The molluscicidal efficacy of mohavingoraj, shaora, haicha, kalmi, bishkatali and nishinda against $I$. exustus could not be compared due to paucity of relevant literature. However, from the study, it was evident that aqueous extract of rakta-karabi exerted rapid action on $I$. exustus than other preparation of that plant. On the other hand, methanol extract of nishinda acted rapidly than other preparation. Therefore, it could be assumed that probably the active ingredient of rakta-karabi which effective against $I$. exustus dissolves more rapidly in water than in ethanol and methanol. But active ingredient of nishinda that acts on I. exustus possibly dissolves rapidly in methanol than in ethanol and water. 
Table 1. In vitro percent mortality of Indoplanorbis exustus exposed to different plant extracts (methanol, ethanol and aqueous)

\begin{tabular}{|c|c|c|c|c|c|c|c|c|c|c|}
\hline \multirow[t]{3}{*}{ Plants } & \multirow{3}{*}{$\begin{array}{l}\text { Conc. } \\
\text { (\%) }\end{array}$} & \multicolumn{9}{|c|}{ Mortality (\%) } \\
\hline & & \multicolumn{3}{|c|}{ Methanol extract } & \multicolumn{3}{|c|}{ Ethanol extract } & \multicolumn{3}{|c|}{ Aqueous extract } \\
\hline & & 6 HAT & 12 HAT & 24 HAT & $6 \mathrm{HAT}$ & 12 HAT & 24 HAT & $6 \mathrm{HAT}$ & 12 HAT & $24 \mathrm{HAT}$ \\
\hline Dhol Kalmi & $\begin{array}{l}1 \\
2 \\
4\end{array}$ & $\begin{array}{l}40^{\mathrm{c}} \\
80^{\mathrm{b}} \\
100^{\mathrm{a}}\end{array}$ & $\begin{array}{l}80^{\mathrm{b}} \\
100^{\mathrm{a}} \\
-\end{array}$ & $\begin{array}{l}100^{\mathrm{a}} \\
100^{\mathrm{a}} \\
-\end{array}$ & $\begin{array}{l}20^{\mathrm{b}} \\
100^{\mathrm{a}} \\
100^{\mathrm{a}}\end{array}$ & $\begin{array}{l}60^{b} \\
- \\
-\end{array}$ & $\begin{array}{l}100^{\mathrm{a}} \\
- \\
-\end{array}$ & $\begin{array}{l}0 \\
0 \\
0\end{array}$ & $\begin{array}{l}0 \\
20^{\mathrm{c}} \\
30^{\mathrm{b}}\end{array}$ & $\begin{array}{l}20^{\mathrm{c}} \\
30^{\mathrm{b}} \\
50^{\mathrm{a}}\end{array}$ \\
\hline Lantana & $\begin{array}{l}1 \\
2 \\
4\end{array}$ & $\begin{array}{l}60^{\mathrm{c}} \\
80^{\mathrm{b}} \\
100^{\mathrm{a}}\end{array}$ & $\begin{array}{l}80^{\mathrm{b}} \\
80^{\mathrm{b}} \\
-\end{array}$ & $\begin{array}{l}100^{\mathrm{a}} \\
100^{\mathrm{a}} \\
-\end{array}$ & $\begin{array}{l}60^{\mathrm{c}} \\
80^{\mathrm{b}} \\
100^{\mathrm{a}}\end{array}$ & $\begin{array}{l}80 \\
80^{b} \\
-\end{array}$ & $\begin{array}{l}100^{\mathrm{a}} \\
100^{\mathrm{a}} \\
-\end{array}$ & $\begin{array}{l}0 \\
0 \\
0\end{array}$ & $\begin{array}{l}20^{\mathrm{c}} \\
30^{\mathrm{b}} \\
80^{\mathrm{b}}\end{array}$ & $\begin{array}{l}80^{\mathrm{b}} \\
100^{\mathrm{a}} \\
100^{\mathrm{a}}\end{array}$ \\
\hline Rakta-karabi & $\begin{array}{l}1 \\
2 \\
4\end{array}$ & $\begin{array}{l}40^{\mathrm{b}} \\
60^{\mathrm{a}} \\
60^{\mathrm{a}}\end{array}$ & $\begin{array}{l}80^{\mathrm{b}} \\
100^{\mathrm{a}} \\
100^{\mathrm{a}}\end{array}$ & $\begin{array}{l}100^{\mathrm{a}} \\
100^{\mathrm{a}} \\
-\end{array}$ & $\begin{array}{l}0 \\
100^{\mathrm{a}} \\
100^{\mathrm{a}}\end{array}$ & $\begin{array}{l}20^{\mathrm{b}} \\
- \\
-\end{array}$ & $\begin{array}{l}100^{\mathrm{a}} \\
- \\
-\end{array}$ & $\begin{array}{l}100^{\mathrm{a}} \\
100^{\mathrm{a}} \\
100^{\mathrm{a}}\end{array}$ & $\begin{array}{l}- \\
- \\
-\end{array}$ & $\begin{array}{l}- \\
- \\
-\end{array}$ \\
\hline Polash & $\begin{array}{l}1 \\
2 \\
4\end{array}$ & $\begin{array}{l}0 \\
20^{\mathrm{b}} \\
40^{\mathrm{a}}\end{array}$ & $\begin{array}{l}20^{\mathrm{b}} \\
40^{\mathrm{b}} \\
80^{\mathrm{a}}\end{array}$ & $\begin{array}{l}20^{\mathrm{b}} \\
40^{\mathrm{b}} \\
80^{\mathrm{a}}\end{array}$ & $\begin{array}{l}20^{\mathrm{c}} \\
40^{\mathrm{b}} \\
60^{\mathrm{a}}\end{array}$ & $\begin{array}{l}- \\
40^{\mathrm{b}} \\
60^{\mathrm{a}}\end{array}$ & $\begin{array}{l}100^{\mathrm{a}} \\
100^{\mathrm{a}} \\
100^{\mathrm{a}}\end{array}$ & $\begin{array}{l}0 \\
0 \\
0\end{array}$ & $\begin{array}{l}0 \\
20^{\mathrm{c}} \\
20^{\mathrm{c}}\end{array}$ & $\begin{array}{l}0 \\
20^{\mathrm{b}} \\
30^{\mathrm{a}}\end{array}$ \\
\hline Mohavingoraj & $\begin{array}{l}1 \\
2 \\
4\end{array}$ & $\begin{array}{l}20^{\mathrm{c}} \\
60^{\mathrm{b}} \\
100^{\mathrm{a}}\end{array}$ & $\begin{array}{l}60^{\mathrm{b}} \\
100^{\mathrm{a}} \\
-\end{array}$ & $\begin{array}{l}100^{\mathrm{a}} \\
100^{\mathrm{a}} \\
100^{\mathrm{a}}\end{array}$ & $\begin{array}{l}80^{\mathrm{b}} \\
100^{\mathrm{a}} \\
100^{\mathrm{a}}\end{array}$ & $\begin{array}{l}80^{b} \\
- \\
-\end{array}$ & $\begin{array}{l}100^{\mathrm{a}} \\
- \\
-\end{array}$ & $\begin{array}{l}40^{\mathrm{c}} \\
60^{\mathrm{b}} \\
90^{\mathrm{a}}\end{array}$ & $\begin{array}{l}60^{\mathrm{b}} \\
90^{\mathrm{a}} \\
100^{\mathrm{a}}\end{array}$ & $\begin{array}{l}60^{\mathrm{b}} \\
100^{\mathrm{a}} \\
-\end{array}$ \\
\hline Nishinda & $\begin{array}{l}1 \\
2 \\
4\end{array}$ & $\begin{array}{l}100^{\mathrm{a}} \\
100^{\mathrm{a}} \\
100^{\mathrm{a}}\end{array}$ & $\begin{array}{l}- \\
- \\
-\end{array}$ & $\begin{array}{l}- \\
- \\
-\end{array}$ & $\begin{array}{l}60^{\mathrm{b}} \\
100^{\mathrm{a}} \\
100^{\mathrm{a}}\end{array}$ & $\begin{array}{l}80^{b} \\
- \\
-\end{array}$ & $\begin{array}{l}100^{\mathrm{a}} \\
- \\
-\end{array}$ & $\begin{array}{l}0 \\
0 \\
0\end{array}$ & $\begin{array}{l}0 \\
0 \\
0\end{array}$ & $\begin{array}{l}0 \\
0 \\
20^{\mathrm{a}}\end{array}$ \\
\hline Kalmi & $\begin{array}{l}1 \\
2 \\
4\end{array}$ & $\begin{array}{l}40^{\mathrm{c}} \\
80^{\mathrm{b}} \\
80^{\mathrm{b}}\end{array}$ & $\begin{array}{l}80^{\mathrm{b}} \\
80^{\mathrm{b}} \\
100^{\mathrm{a}}\end{array}$ & $\begin{array}{l}60^{\mathrm{d}} \\
100^{\mathrm{a}} \\
-\end{array}$ & $\begin{array}{l}20^{\mathrm{b}} \\
60^{\mathrm{d}} \\
100^{\mathrm{a}}\end{array}$ & $\begin{array}{l}60^{\mathrm{d}} \\
100^{\mathrm{a}} \\
-\end{array}$ & $\begin{array}{l}40^{\mathrm{c}} \\
100^{\mathrm{a}} \\
-\end{array}$ & $\begin{array}{l}40^{\mathrm{c}} \\
80^{\mathrm{b}} \\
100^{\mathrm{a}}\end{array}$ & $\begin{array}{l}80^{\mathrm{b}} \\
100^{\mathrm{a}} \\
-\end{array}$ & $\begin{array}{l}100^{\mathrm{a}} \\
- \\
-\end{array}$ \\
\hline Bishkatali & $\begin{array}{l}1 \\
2 \\
4\end{array}$ & $\begin{array}{l}40^{\mathrm{c}} \\
60 \\
80^{\mathrm{b}}\end{array}$ & $\begin{array}{l}60 \\
80^{\mathrm{b}} \\
100^{\mathrm{a}}\end{array}$ & $\begin{array}{l}100^{\mathrm{a}} \\
100^{\mathrm{a}}\end{array}$ & $\begin{array}{l}60^{\mathrm{b}} \\
80 \\
100\end{array}$ & $\begin{array}{l}80 \\
100\end{array}$ & $\begin{array}{l}100^{\mathrm{a}} \\
100^{\mathrm{a}}\end{array}$ & $\begin{array}{l}20^{\mathrm{c}} \\
40 \\
60^{\mathrm{b}}\end{array}$ & $\begin{array}{l}40 \\
60^{\mathrm{b}} \\
80^{\mathrm{a}}\end{array}$ & $\begin{array}{l}60^{\mathrm{a}} \\
80^{\mathrm{a}} \\
100^{\mathrm{a}}\end{array}$ \\
\hline Shaora & $\begin{array}{l}1 \\
2\end{array}$ & $\begin{array}{l}0 \\
0\end{array}$ & $\begin{array}{l}0 \\
0\end{array}$ & $\begin{array}{l}0 \\
0^{\mathrm{b}}\end{array}$ & $\begin{array}{l}0 \\
0\end{array}$ & $\begin{array}{l}0 \\
0\end{array}$ & $\begin{array}{l}0 \\
0\end{array}$ & $\begin{array}{l}0 \\
0\end{array}$ & $\begin{array}{l}0 \\
0\end{array}$ & $\begin{array}{l}0 \\
0\end{array}$ \\
\hline & 4 & 0 & 0 & $20^{\mathrm{a}}$ & 0 & 0 & $40^{\mathrm{a}}$ & 0 & 0 & 0 \\
\hline Haicha & $\begin{array}{l}1 \\
2 \\
4\end{array}$ & $\begin{array}{l}60^{\mathrm{b}} \\
80 \\
100^{\mathrm{a}}\end{array}$ & $\begin{array}{l}60^{\mathrm{b}} \\
80 \\
-\end{array}$ & $\begin{array}{l}100^{\mathrm{a}} \\
100^{\mathrm{a}} \\
-\end{array}$ & $\begin{array}{l}60^{\mathrm{c}} \\
100^{\mathrm{b}} \\
100^{\mathrm{a}}\end{array}$ & $\begin{array}{l}100^{\mathrm{b}} \\
- \\
-\end{array}$ & $\begin{array}{l}100^{\mathrm{a}} \\
- \\
-\end{array}$ & $\begin{array}{l}20^{\mathrm{d}} \\
40^{\mathrm{c}} \\
40^{\mathrm{c}}\end{array}$ & $\begin{array}{l}20^{\mathrm{c}} \\
80^{\mathrm{b}} \\
80\end{array}$ & $\begin{array}{l}80^{\mathrm{b}} \\
80 \\
100^{\mathrm{a}}\end{array}$ \\
\hline Control & - & - & & - & - & & - & - & & - \\
\hline
\end{tabular}

HAT $=$ Hours after treatment, Values having different superscripts in the same column were statistically significant $(\mathrm{p}<0.01)$

\section{Effects on L. auricularia}

Methanol extract of mohavingoraj was most effective against L. auricularia as it caused $100 \%$ mortality in all concentrations applied (1, 2 and 4\%) within 6 hours of treatment. Nishinda and haicha at higher concentration (2 and $4 \%$ ) killed $100 \%$ of the snails by 6 hours post-treatment. On the other hand, 1, 2 and $4 \%$ ethanol extracts of lantana, haicha and nishinda killed 100\% snails tested, by 6 hours. But rakta-karabi, bishkatali and dhol kalmi at higher concentrations ( 2 and 4\%) caused death of 100\% snails within 6 hours of treatment. Aqueous extract of rakt-karabi exerted the highest efficacy (100\%) against $L$. auricularia. Two (2) and 4\% water extract of lantana, kalmi, haicha and mohavingoraj killed $100 \%$ snails within 24 hours (Table 2). These findings could not be compared and contrasted due to paucity of relevant literature. However, result of the present work implies that ethanol extract of lantana, nishinda and haicha acted rapidly on L. auricularia. But methanol extract of mohavingoraj exerted action within very short time. From this observation it can be concluded that active ingredient of lantana, nishinda and haicha, which is active against $L$. auricularia, dissolves rapidly in ethanol. But that of mohavingoraj that is effective against $L$. auricularia dissolve rapidly in methanol. 
Indigenous plants and control of vector snails

Table 2. In vitro percent mortality of Lymnea auricularia exposed to different plant extracts (methanol, ethanol and aqueous)

\begin{tabular}{|c|c|c|c|c|c|c|c|c|c|c|}
\hline \multirow[t]{3}{*}{ Plants } & \multirow{3}{*}{$\begin{array}{l}\text { Conc. } \\
\text { (\%) }\end{array}$} & \multicolumn{9}{|c|}{ Mortality (\%) } \\
\hline & & \multicolumn{3}{|c|}{ Methanol } & \multicolumn{3}{|l|}{ Ethanol } & \multicolumn{3}{|c|}{ Aqueous } \\
\hline & & 6 HAT & 12 HAT & 24 HAT & $6 \mathrm{HAT}$ & $12 \mathrm{HAT}$ & $24 \mathrm{HAT}$ & 6 HAT & $12 \mathrm{HAT}$ & $24 \mathrm{HAT}$ \\
\hline \multirow[t]{3}{*}{ Dhol Kalmi } & 1 & $40^{\mathrm{C}}$ & $60^{\mathrm{b}}$ & $100^{\mathrm{a}}$ & $20^{\mathrm{b}}$ & 60 & $100^{\mathrm{a}}$ & 0 & 0 & $30^{\mathrm{C}}$ \\
\hline & 2 & $60^{\mathrm{b}}$ & $80^{\mathrm{a}}$ & $100^{\mathrm{a}}$ & $100^{\mathrm{a}}$ & - & - & 0 & $30^{\mathrm{c}}$ & $40^{\mathrm{b}}$ \\
\hline & 4 & $80^{\mathrm{a}}$ & $100^{\mathrm{a}}$ & - & $100^{\mathrm{a}}$ & - & - & 0 & 40 & $50^{\mathrm{a}}$ \\
\hline \multirow{3}{*}{ Lantana } & 1 & $40^{\mathrm{C}}$ & $60^{\mathrm{b}}$ & $100^{\mathrm{a}}$ & $100^{\mathrm{a}}$ & - & - & 0 & 30 & $80^{\mathrm{b}}$ \\
\hline & 2 & $60^{\mathrm{b}}$ & $100^{\mathrm{a}}$ & - & $100^{\mathrm{a}}$ & - & - & 0 & 40 & $100^{\mathrm{a}}$ \\
\hline & 4 & $100^{\mathrm{a}}$ & - & - & $100^{\mathrm{a}}$ & - & - & 0 & 50 & $100^{\mathrm{a}}$ \\
\hline \multirow[t]{3}{*}{ Rakta karabi } & 1 & 0 & 0 & $100^{\mathrm{a}}$ & $80^{\mathrm{b}}$ & $100^{\mathrm{a}}$ & - & $100^{\mathrm{a}}$ & - & - \\
\hline & 2 & $60^{\mathrm{a}}$ & $60^{\mathrm{b}}$ & $100^{\mathrm{a}}$ & $100^{\mathrm{a}}$ & - & - & $100^{\mathrm{a}}$ & - & - \\
\hline & 4 & $60^{\mathrm{a}}$ & $80^{c}$ & $100^{\mathrm{a}}$ & $100^{\mathrm{a}}$ & - & - & $100^{\mathrm{a}}$ & - & - \\
\hline \multirow[t]{3}{*}{ Polash } & 1 & 0 & $20^{\mathrm{b}}$ & $100^{\mathrm{a}}$ & 0 & $40^{\mathrm{b}}$ & $100^{\mathrm{a}}$ & 0 & 0 & $10^{\mathrm{c}}$ \\
\hline & 2 & $20^{\mathrm{b}}$ & $40^{\mathrm{a}}$ & $100^{\mathrm{a}}$ & 0 & $40^{\mathrm{b}}$ & $100^{\mathrm{a}}$ & 0 & $10^{\mathrm{c}}$ & $30^{\mathrm{b}}$ \\
\hline & 4 & $40^{\mathrm{a}}$ & 50 & $100^{\mathrm{a}}$ & 0 & $80^{\mathrm{c}}$ & $100^{\mathrm{a}}$ & 0 & $30^{\mathrm{b}}$ & $50^{\mathrm{a}}$ \\
\hline \multirow[t]{3}{*}{ Mohavingoraj } & 1 & $100^{\mathrm{a}}$ & - & - & $40^{\mathrm{c}}$ & $80^{\mathrm{b}}$ & $80^{\mathrm{b}}$ & 40 & $60^{\mathrm{b}}$ & $80^{\mathrm{b}}$ \\
\hline & 2 & $100^{\mathrm{a}}$ & - & - & $80^{\mathrm{b}}$ & $80^{b}$ & $100^{\mathrm{a}}$ & $60^{\mathrm{b}}$ & $80^{b}$ & $100^{\mathrm{a}}$ \\
\hline & 4 & $100^{\mathrm{a}}$ & - & - & $100^{\mathrm{a}}$ & - & - & $70^{\mathrm{a}}$ & $100^{\mathrm{a}}$ & \\
\hline \multirow[t]{3}{*}{ Nishinda } & 1 & $80^{\mathrm{b}}$ & $80^{\mathrm{b}}$ & $100^{\mathrm{a}}$ & $100^{\mathrm{a}}$ & - & - & 0 & 0 & 0 \\
\hline & 2 & $100^{\mathrm{a}}$ & - & - & $100^{\mathrm{a}}$ & - & - & 0 & 0 & 0 \\
\hline & 4 & $100^{\mathrm{a}}$ & - & - & $100^{\mathrm{a}}$ & - & - & 0 & 0 & $40^{\mathrm{a}}$ \\
\hline \multirow[t]{3}{*}{ Kalmi } & 1 & $60^{\mathrm{c}}$ & $80^{\mathrm{b}}$ & $80^{\mathrm{b}}$ & $40^{\mathrm{c}}$ & $80^{\mathrm{b}}$ & $40^{\mathrm{c}}$ & $80^{\mathrm{b}}$ & $80^{\mathrm{b}}$ & $80^{\mathrm{b}}$ \\
\hline & 2 & $80^{\mathrm{b}}$ & $80^{\mathrm{b}}$ & $80^{\mathrm{b}}$ & $80^{\mathrm{b}}$ & $80^{\mathrm{b}}$ & $100^{\mathrm{a}}$ & $80^{\mathrm{b}}$ & $100^{\mathrm{a}}$ & - \\
\hline & 4 & $80^{\mathrm{b}}$ & $100^{\mathrm{a}}$ & - & $100^{\mathrm{a}}$ & - & - & $100^{\mathrm{a}}$ & - & - \\
\hline \multirow[t]{3}{*}{ Bishkatali } & 1 & $60^{\mathrm{c}}$ & $60 c$ & $100^{\mathrm{a}}$ & $60^{\mathrm{b}}$ & - & $100^{\mathrm{a}}$ & $20^{\mathrm{C}}$ & $80^{\mathrm{a}}$ & $60^{\mathrm{a}}$ \\
\hline & 2 & $60 c$ & $80^{\mathrm{b}}$ & - & $100^{\mathrm{a}}$ & - & - & $80^{\mathrm{a}}$ & $100^{\mathrm{a}}$ & $80^{\mathrm{a}}$ \\
\hline & 4 & $80^{\mathrm{b}}$ & $100^{\mathrm{a}}$ & & $100^{\mathrm{a}}$ & - & - & $100^{\mathrm{a}}$ & - & - \\
\hline \multirow[t]{3}{*}{ Shaora } & 1 & 0 & 0 & 0 & 0 & 0 & 0 & 0 & 0 & 0 \\
\hline & 2 & 0 & 0 & 0 & 0 & 0 & 0 & 0 & 0 & 0 \\
\hline & 4 & 0 & 0 & 0 & 0 & 0 & $40^{\mathrm{a}}$ & 0 & 0 & 0 \\
\hline \multirow[t]{3}{*}{ Haicha } & 1 & $60^{\mathrm{b}}$ & $100^{\mathrm{a}}$ & - & $100^{\mathrm{a}}$ & - & - & $20^{\mathrm{d}}$ & $40^{\mathrm{c}}$ & $80^{\mathrm{b}}$ \\
\hline & 2 & $100^{\mathrm{a}}$ & - & - & $100^{\mathrm{b}}$ & - & - & $40^{\mathrm{C}}$ & $40^{\mathrm{C}}$ & $100^{\mathrm{a}}$ \\
\hline & 4 & $100^{\mathrm{a}}$ & - & - & $100^{\mathrm{a}}$ & - & - & $40^{\mathrm{C}}$ & $100^{\mathrm{a}}$ & - \\
\hline Control & - & - & - & - & - & & - & - & & - \\
\hline
\end{tabular}

HAT $=$ Hours after treatment, Values having different superscripts in the same column were statistically significant $(\mathrm{p}<0.01)$

\section{Effects on L. luteola}

One (1), 2 and $4 \%$ methanol extract of mohavingoraj showed the highest efficacy (100\%) against L. luteola within 6 hours. But methanol extract of nishinda and kalmi killed 100\% snails at 2 and 4\% concentrations by 6 hours. Ethanol extract of lantana showed the highest efficacy (100\%) in all concentrations used (1, 2 and $4 \%$ ) by 6 hours against $L$. luteola. Two per cent and 4\% ethanol extract of mohavingoraj and nishinda killed $100 \%$ snails within 6 hours of treatment. In case of aqueous extract, the highest (100\%) molluscicidal activity was observed at 1,2 and $4 \%$ concentrations of rakta-karabi, 2 and $4 \%$ haicha and $4 \%$ bishkatali followed by mohavingoraj at $4 \%$ concentration (90\%) within 6 hours (Table 3). These findings could not be compared due to lack of relevant literature. However, the molluscicidal activity of some plants has been tested against other lymnaeid species. Kumar and Singh (2006) studied the molluscicidal activity of dried root latex powder of Ferula asafoetida, flower-bud powder of Syzygium aromaticum and seed powder of Carum carvi against the snail Lymnaea acuminata. 
The molluscicidal activity of all the three plant products was found to be both time and concentration dependent. Ethanol extract was more toxic than other organic extracts. Singh and Singh (2005) reported the mortality caused by the aqueous extract and latex of Thevetia peruviana against harmful freshwater snails, Lymnaea acuminata. Latex of the plant at high doses were also lethal to freshwater fish Channa punctatus, but within 24 hours $L_{90}$ of snail $L$. acuminata did not cause any mortality to fishes in a mixed population of snail and fish.

Table 3. In vitro percent mortality of Lymnea luteola exposed to different plant extracts (methanol, ethanol and aqueous)

\begin{tabular}{|c|c|c|c|c|c|c|c|c|c|c|}
\hline \multirow[t]{3}{*}{ Plants } & \multirow{3}{*}{$\begin{array}{l}\text { Conc. } \\
\text { (\%) }\end{array}$} & \multicolumn{9}{|c|}{ Mortality (\%) } \\
\hline & & \multicolumn{3}{|c|}{ Methanol } & \multicolumn{3}{|l|}{ Ethanol } & \multicolumn{3}{|c|}{ Aqueous } \\
\hline & & 6 HAT & 12 HAT & 24 HAT & 6 HAT & 12 HAT & 24 HAT & 6 HAT & 12 HAT & 24 HAT \\
\hline$\overline{\text { Dhol Kalmi }}$ & $\begin{array}{l}1 \\
2 \\
4\end{array}$ & $\begin{array}{l}20^{\mathrm{b}} \\
60^{\mathrm{a}} \\
60^{\mathrm{a}}\end{array}$ & $\begin{array}{l}60^{\mathrm{a}} \\
60^{\mathrm{a}} \\
100^{\mathrm{a}}\end{array}$ & $\begin{array}{l}100^{\mathrm{a}} \\
100^{\mathrm{a}}\end{array}$ & $\begin{array}{l}20^{\mathrm{b}} \\
20^{\mathrm{b}} \\
100^{\mathrm{a}}\end{array}$ & $\begin{array}{l}20^{\mathrm{b}} \\
100^{\mathrm{a}} \\
-\end{array}$ & $\begin{array}{l}100^{\mathrm{a}} \\
100^{\mathrm{a}} \\
-\end{array}$ & $\begin{array}{l}0 \\
0 \\
0\end{array}$ & $\begin{array}{l}0 \\
40^{\mathrm{c}} \\
50^{\mathrm{b}}\end{array}$ & $\begin{array}{l}40^{\mathrm{c}} \\
50^{\mathrm{b}} \\
70^{\mathrm{a}}\end{array}$ \\
\hline Lantana & $\begin{array}{l}1 \\
2 \\
4\end{array}$ & $\begin{array}{l}20^{\mathrm{c}} \\
40^{\mathrm{b}} \\
100^{\mathrm{a}}\end{array}$ & $\begin{array}{l}40^{\mathrm{b}} \\
100^{\mathrm{a}} \\
-\end{array}$ & $\begin{array}{l}100^{\mathrm{a}} \\
100^{\mathrm{a}} \\
-\end{array}$ & $\begin{array}{l}100^{\mathrm{a}} \\
100^{\mathrm{a}} \\
100^{\mathrm{a}}\end{array}$ & $\begin{array}{l}- \\
-\end{array}$ & $\begin{array}{l}- \\
-\end{array}$ & $\begin{array}{l}0 \\
0 \\
0\end{array}$ & $\begin{array}{l}0 \\
0 \\
40^{\mathrm{b}}\end{array}$ & $\begin{array}{l}20^{\mathrm{c}} \\
40^{\mathrm{b}} \\
60^{\mathrm{a}}\end{array}$ \\
\hline Rakta-karabi & $\begin{array}{l}1 \\
2 \\
4\end{array}$ & $\begin{array}{l}0 \\
0 \\
20^{\mathrm{a}}\end{array}$ & $\begin{array}{l}0 \\
0 \\
60\end{array}$ & $\begin{array}{l}100^{\mathrm{a}} \\
100^{\mathrm{a}} \\
100^{\mathrm{a}}\end{array}$ & $\begin{array}{l}40^{\mathrm{c}} \\
80^{\mathrm{b}} \\
100^{\mathrm{a}}\end{array}$ & $\begin{array}{l}80^{\mathrm{b}} \\
- \\
-\end{array}$ & $\begin{array}{l}100^{\mathrm{a}} \\
100^{\mathrm{a}} \\
-\end{array}$ & $\begin{array}{l}100^{\mathrm{a}} \\
100^{\mathrm{a}} \\
100^{\mathrm{a}}\end{array}$ & $\begin{array}{l}- \\
-\end{array}$ & $\begin{array}{l}- \\
-\end{array}$ \\
\hline Polash & $\begin{array}{l}1 \\
2 \\
4\end{array}$ & $\begin{array}{l}0 \\
0 \\
0\end{array}$ & $\begin{array}{l}0 \\
0 \\
40\end{array}$ & $\begin{array}{l}40^{\mathrm{b}} \\
40^{\mathrm{b}} \\
60^{\mathrm{a}}\end{array}$ & $\begin{array}{l}0 \\
20^{\mathrm{b}} \\
40^{\mathrm{a}}\end{array}$ & $\begin{array}{l}40^{\mathrm{a}} \\
40^{\mathrm{a}} \\
100^{\mathrm{a}}\end{array}$ & $\begin{array}{l}100^{\mathrm{a}} \\
100^{\mathrm{a}} \\
-\end{array}$ & $\begin{array}{l}0 \\
0 \\
0\end{array}$ & $\begin{array}{l}0 \\
20^{\mathrm{b}} \\
50^{\mathrm{a}}\end{array}$ & $\begin{array}{l}10^{\mathrm{c}} \\
20^{\mathrm{b}} \\
50^{\mathrm{a}}\end{array}$ \\
\hline Mohavingoraj & $\begin{array}{l}1 \\
2 \\
4\end{array}$ & $\begin{array}{l}100^{\mathrm{a}} \\
100^{\mathrm{a}} \\
100^{\mathrm{a}}\end{array}$ & - & $\begin{array}{l}- \\
-\end{array}$ & $\begin{array}{l}80^{\mathrm{b}} \\
100^{\mathrm{a}} \\
100^{\mathrm{a}}\end{array}$ & $\begin{array}{l}60 \\
- \\
-\end{array}$ & $\begin{array}{l}100^{\mathrm{a}} \\
- \\
-\end{array}$ & $\begin{array}{l}60^{\mathrm{c}} \\
70^{\mathrm{b}} \\
90^{\mathrm{a}}\end{array}$ & $\begin{array}{l}80^{\mathrm{b}} \\
90^{\mathrm{a}} \\
100^{\mathrm{a}}\end{array}$ & $\begin{array}{l}80^{\mathrm{b}} \\
100^{\mathrm{a}}\end{array}$ \\
\hline Nishinda & $\begin{array}{l}1 \\
2 \\
4\end{array}$ & $\begin{array}{l}80^{\mathrm{b}} \\
100^{\mathrm{a}} \\
100^{\mathrm{a}}\end{array}$ & $\begin{array}{l}80^{\mathrm{b}} \\
- \\
-\end{array}$ & $\begin{array}{l}100^{\mathrm{a}} \\
- \\
-\end{array}$ & $\begin{array}{l}60^{\mathrm{b}} \\
100^{\mathrm{a}} \\
100^{\mathrm{a}}\end{array}$ & $\begin{array}{l}80 \\
90 \\
-\end{array}$ & $\begin{array}{l}100^{\mathrm{a}} \\
- \\
-\end{array}$ & $\begin{array}{l}0 \\
0 \\
0\end{array}$ & $\begin{array}{l}0 \\
0 \\
0\end{array}$ & $\begin{array}{l}0 \\
0 \\
20^{\text {a }}\end{array}$ \\
\hline Kalmi & $\begin{array}{l}1 \\
2 \\
4\end{array}$ & $\begin{array}{l}60^{\mathrm{c}} \\
100^{\mathrm{a}} \\
100^{\mathrm{a}}\end{array}$ & $\begin{array}{l}80 \\
- \\
-\end{array}$ & $\begin{array}{l}80^{b} \\
- \\
-\end{array}$ & $\begin{array}{l}40^{\mathrm{c}} \\
80^{\mathrm{b}} \\
80^{\mathrm{b}}\end{array}$ & $\begin{array}{l}80^{\mathrm{b}} \\
80^{\mathrm{b}} \\
100^{\mathrm{a}}\end{array}$ & $\begin{array}{l}40^{\mathrm{c}} \\
100^{\mathrm{a}}\end{array}$ & $\begin{array}{l}80^{\mathrm{b}} \\
80^{\mathrm{b}} \\
80^{\mathrm{b}}\end{array}$ & $\begin{array}{l}80^{\mathrm{b}} \\
80^{\mathrm{b}} \\
100^{\mathrm{a}}\end{array}$ & $\begin{array}{l}80^{\mathrm{b}} \\
100^{\mathrm{a}}\end{array}$ \\
\hline Bishkatali & 1 & $60^{\mathrm{c}}$ & $70^{\mathrm{c}}$ & $100^{\mathrm{a}}$ & $60^{\mathrm{b}}$ & $80^{\mathrm{b}}$ & $100^{\mathrm{a}}$ & $20^{c}$ & $80^{\mathrm{a}}$ & $60^{\mathrm{a}}$ \\
\hline & $\begin{array}{l}2 \\
4\end{array}$ & $\begin{array}{l}60^{\mathrm{c}} \\
80^{\mathrm{b}}\end{array}$ & $\begin{array}{l}80^{\mathrm{b}} \\
100^{\mathrm{a}}\end{array}$ & $100^{\mathrm{a}}$ & $\begin{array}{l}80^{\mathrm{b}} \\
100^{\mathrm{a}}\end{array}$ & $\begin{array}{l}100^{\mathrm{a}} \\
-\end{array}$ & $\begin{array}{l}- \\
-\end{array}$ & $\begin{array}{l}80^{\mathrm{a}} \\
100^{\mathrm{a}}\end{array}$ & $\begin{array}{l}100^{\mathrm{a}} \\
-\end{array}$ & - \\
\hline Shaora & $\begin{array}{l}1 \\
2 \\
4\end{array}$ & $\begin{array}{l}0 \\
0 \\
0\end{array}$ & $\begin{array}{l}0 \\
0 \\
0\end{array}$ & $\begin{array}{l}0 \\
0 \\
0\end{array}$ & $\begin{array}{l}0 \\
0 \\
0\end{array}$ & $\begin{array}{l}0 \\
0 \\
0\end{array}$ & $\begin{array}{l}0 \\
0 \\
40^{\mathrm{a}}\end{array}$ & $\begin{array}{l}0 \\
0 \\
0\end{array}$ & $\begin{array}{l}0 \\
0 \\
0\end{array}$ & $\begin{array}{l}0 \\
0 \\
0\end{array}$ \\
\hline Haicha & $\begin{array}{l}1 \\
2 \\
4\end{array}$ & $\begin{array}{l}60^{\mathrm{b}} \\
80^{\mathrm{b}} \\
100^{\mathrm{a}}\end{array}$ & $\begin{array}{l}80^{\mathrm{b}} \\
100^{\mathrm{a}}\end{array}$ & $\begin{array}{l}100^{\mathrm{a}} \\
- \\
-\end{array}$ & $\begin{array}{l}80^{\mathrm{b}} \\
80^{\mathrm{b}} \\
100^{\mathrm{a}}\end{array}$ & $\begin{array}{l}100^{\mathrm{a}} \\
100^{\mathrm{a}} \\
-\end{array}$ & - & $\begin{array}{l}20^{\mathrm{d}} \\
100^{\mathrm{a}} \\
100^{\mathrm{a}}\end{array}$ & $\begin{array}{l}40 \\
- \\
-\end{array}$ & $\begin{array}{l}80^{b} \\
- \\
-\end{array}$ \\
\hline Control & - & - & & - & - & & - & - & & - \\
\hline
\end{tabular}

HAT = Hours after treatment, Values having different superscripts in the same column were statistically significant $(\mathrm{p}<0.01)$ 
Indigenous plants and control of vector snails

Table 4. Minimum concentration and $\mathrm{LD}_{50}$ of some indigenous plants in snail and their effects on fish (Gutum, Letidocephalus guntea, Family: Cobitidae)

\begin{tabular}{|llll|}
\hline Plants & $\begin{array}{l}\text { Min.C } \\
\text { (\%) with dust } \\
(24 \mathrm{HAT})\end{array}$ & $\begin{array}{l}\mathrm{LD}_{50}(\mathrm{Dust}) \mathrm{mg} / \mathrm{L} \\
(24 \mathrm{HAT})\end{array}$ & $\begin{array}{l}\text { Mortality (\%), Fish at } \\
\text { Min.C }_{\text {s100 }} \\
(24 \text { HAT) }\end{array}$ \\
\hline Dhol Kalmi & 0.25 & 661 & 40 \\
Lantana & 0.06 & 234 & 0 \\
Rakta-karabi & 0.008 & 29.25 & 100 \\
Polash & 0.5 & 2262 & 100 \\
Mohavingoraj & 0.5 & 1326 & 100 \\
Nishinda & 0.5 & 1092 & 100 \\
Kalmi & 0.25 & 154.685 & 0 \\
Bishkatali & 0.125 & 46.875 & 100 \\
Shaora & 1 & 453.125 & 0 \\
Haicha & 0.03 & 14.3 & 0 \\
Control & - & - & - \\
\hline
\end{tabular}

HAT $=$ Hours after treatment, Min. $\mathrm{C}_{\mathrm{s} 100}=$ Minimum concentration which killed $100 \%$ snails by 24 hours, $\mathrm{LD}_{50}=$ Lethal dose 50.

\section{Toxicity study of tested plants dust on fish}

In this study, $\mathrm{LD}_{50}$, Min. $\mathrm{C}_{\mathrm{s} 100}$ and mortality percentage of fish (Gutum, Letidocephalus guntea, Family: Cobitidae) were determined using dust of the tested plants. $\mathrm{LD}_{50}$ was minimum in case of haicha (14.3mg/L) followed by rakta-karabi $(29.25 \mathrm{mg} / \mathrm{L})$, bishkatali $(46.88 \mathrm{mg} / \mathrm{L})$, kalmi $(154.69 \mathrm{mg} / \mathrm{L})$ etc. Min. $\mathrm{C}_{\mathrm{s} 100}$ was minimum in case of rakta-karabi $(0.008 \%)$ followed by haicha $(0.03 \%)$, lantana $(0.06 \%)$, dholkalmi $(0.25 \%)$ and kalmi (0.25\%). Among these plants rakta-karabi, polash, mohavingoraj, nishinda and bishkatali were highly toxic for fish, which killed 100\% fish within 24 hours. But lantana, haicha and kalmi had no lethal effect on fish. Shaora also had no toxic effect on fish but its both $\mathrm{LD}_{50}(453.13 \mathrm{mg} / \mathrm{L})$ and Min. $\mathrm{C}_{\mathrm{s} 100}(1 \%)$ were very high (Table 4). $\mathrm{LD}_{50}$ of $N$. indicum was also caculated by Wang et al (2006). They reported the $\mathrm{LD}_{50}$ of water extract of $N$. indicum as low as $13.2 \mathrm{mg} / \mathrm{L}$. This variation in between the present and previous studies might be due to the variation in the method of study. In the present study the effects of only methanol and/or ethanol (without mixing any plant extracts) were not studied. So, the justification of the results was done by determining the $L_{50}$ by using dust of each plant which is the crudest form of herbal preparation and probably contain relatively less amount of active ingredients than water, methanol and ethanol extracts. Despite of this limitation, dust is comparatively easy to prepare and apply especially in field level. Therefore, from the present study, it can be concluded that dust of haicha, lantana and kalmi may be used @ 0.03\%,0.06\% and 0.25\% respectively to control snails.

\section{ACKNOWLEDGEMENT}

The authors thankfully acknowledge the financial support of University Grants Commission (UGC) of Bangladesh to conduct the research work.

\section{REFERENCES}

1. Amrita S, Singh DK, and Singh A (2001). Molluscicidal activity of Lawsonia inermis and its binary and tertiary combinations with other plant derived molluscicides. Indian Journal of Experimental Biology 39:263-268. 
2. Kumar P and Singh DK (2006). Molluscicidal activity of Ferula asafoetida, Syzygium aromaticum and Carum carvi and their active components against the snail Lymnaea acuminata. Chemosphere 63:1568-1574.

3. Malek EA and Cheng TC (1974). Medical and Economic Malacology, Academic Press Inc. (London Ltd.) pp. 285-319.

4. Mostafa BB, el-Deeb FA, Ismail NM and el-Said KM (2005a). Impact of certain plants and synthetic molluscicides on some fresh water snails and fish. Journal of Egyptian Society of Parasitology 35:989-1007.

5. Pal S, Koley KM and Pathak AK (2002). Molluscicidal activity of Azadirachta indica leaves and Lantana camara leaves against Indoplanorbis exustus. Indian Journal of Animal Science 72:561-562.

6. Singh A and Singh DK (2001). Molluscicidal activity of Lawsonia inermis and its binary and tertiary combinations with other plant derived molluscicides. Indian Journal of Experimental Biology 39:263-268.

7. $\quad$ Singh A and Singh SK (2005). Molluscicidal evaluation of three common plants from India. Fitoterapia 76:747-751.

8. Singh SK, Yadav RP, Tiwari S and Singh A (2005). Toxic effect of stem bark and leaf of Euphorbia hirta plant against freshwater vector snail Lymnaea acuminata. Chemosphere 59:263-270.

9. Steel RGD and Torrie JH (1980). Principles and Procedures of Statistics, McGrow Hill Book Co. Inc., USA.

10. Sultana T, Sahiduzzaman M, Alim MA, Islam MK and Mondal MMH (2004). In vitro screening of some indigenous medicinal plants against Indoplanorbis exustus (Gastropoda: Planorbidae). Progressive Agriculture 15: 41-46.

11. Wang H, Cai WM, Wang WX and Yang JM (2006) Molluscicidal activity of Nerium indicum Mill, Pterocarya stenoptera DC, and Rumex japonicum Houtt on Oncomelania hupensis. Biomedical and Environmental Science 19: 245-248. 